\title{
Carcinoma a cellule squamose nodulare multifocale delle regioni zigomatiche e destruente del collo in una grande anziana con sindrome demenziale
}

\section{Destructive, multifocal squamous-cell carcinoma nodules on the cheecks and neck of an elderly woman with a dementia syndrome}

\section{Norma Marigliano ${ }^{a}$, Manuela Colosimo ${ }^{a}$, Marcella Flocco ${ }^{a}$, Francesca Mazzei ${ }^{a}$, Maria V. Sirianni ${ }^{b}$, Domenico Galasso ${ }^{c, *}$, Luigi Tucci $^{d}$, Francesco Abbonante ${ }^{e}$, Giovanni Ruotolo ${ }^{f}$}

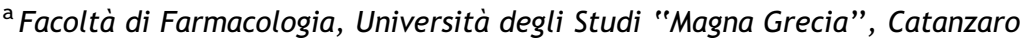

${ }^{\mathrm{b}}$ Psicologa, RSA Madonna di Porto, Catanzaro

"Direttore Emerito di medicina Interna, AO "Pugliese-Ciaccio", Catanzaro

"Direttore SOC Anatomia Patologica, AO "Pugliese-Ciaccio", Catanzaro

"Direttore SOC Chirurgia Plastica, AO "Pugliese-Ciaccio", Catanzaro

"Direttore SOC Geriatria, AO "Pugliese-Ciaccio", Catanzaro
}

Ricevuto il 14 luglio 2009; accettato il 5 giugno 2010

disponibile online il 10 luglio 2010

\section{KEYWORDS}

Multifocal squamous-cell carcinoma;

Dementia syndrome;

Histology;

Outcome.

\begin{abstract}
Summary
Introduction: Squamous-cell carcinoma is the second most common form of skin cancer. It can develop on normal skin, actinic keratoses, leukoplakia, and burn scars. The tumor is characterized by remarkable variability at the macroscopic and histopathologic levels.

Case report: A 93-year-old woman was admitted to nursing home with a diagnosis of dementia syndrome and squamous cell nodular carcinoma on cheeks and neck region. The physical examination revealed firm, black excrescences with irregular surfaces over both cheekbones, which were roughly the size of hazelnuts. Similar nodules were present with ulcers on other areas of the face. The patient was admitted to the day hospital twice for wide excision of the tumors in the zygomatic region. Later, the neck tumors were removed, and the wound was repaired with a rotation flap after careful control of bleeding. For the latter surgery, the patient was hospitalized for a few days in a geriatric ward to ensure optimal medical care and psychological support. The histological examination revealed ulcerated, well-differentiated squamous-cell carcinoma that
\end{abstract}

\footnotetext{
* Corrispondenza: v.le Brutium 46/A - 88100 Catanzaro.

E-mail: domenico.galasso@libero.it (D. Galasso).
} 
extended down to the subcutaneous layer. Shortly after surgery, she returned to the nursing home to resume rehabilitation and group therapy.

(C) 2010 Elsevier Srl. All rights reserved.

\section{Introduzione}

Il carcinoma squamocellulare è il secondo tipo più comune di neoplasia cutanea; può insorgere sia su cute normale sia su aree di preesistente cheratosi attinica, di leucoplachia o su cicatrici da ustioni [1,2]. Il tumore presenta notevole variabilità macroscopica; può esordire come papula o placca eritematosa, coperta da squame o croste, ed evolvere in lesione nodulare a superficie verrucosa, o ulcerarsi infiltrando i tessuti sottostanti. Notevole è anche la variabilità istopatologica [3,4]. Esistono vari gradi di differenziazione: ben differenziato, moderatamente differenziato, poco differenziato. Limitata è la corrispondenza fra grado di differenziazione e comportamento clinico. Nella grande maggioranza dei casi è tipicamente infiltrante già nelle sue manifestazioni iniziali [5]. La prognosi risente del grado di infiltrazione (margini espansivi, margini infiltrativi) e della stadiazione patologica, espressa nelle categorie TNM.

\section{Caso clinico}

Donna, anni 93, ricoverata per una sindrome demenziale. Presenti vegetazioni a carico delle regioni zigomatiche, di colorito nerastro, a superficie irregolare, di consistenza dura, delle dimensioni di una nocciola. Altre aree in parte ulcerate, in parte nodulari, con le stesse caratteristiche ( fig. 1).

Anemia normocromica carenziale: emoglobina 9,2\%; globuli rossi 2.760.000; MCV 96; globuli bianchi 3.700 (neutrofili 65,9\%; linfociti $21,6 \%$; monociti $8,5 \%$; eosinofili $1,3 \%$; basofili 0,1\%); piastrine 271.000. VES 32 .

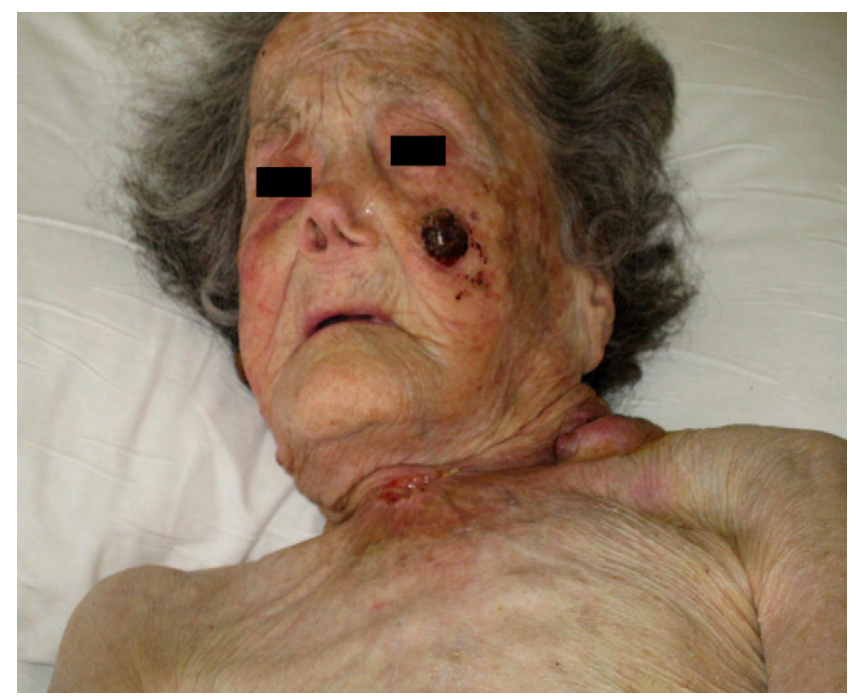

Figura 1 Carcinoma cutaneo a focolai multipli (regione zigomatica bilateralmente e del collo).
Normali gli altri esami ematochimici: INR 0,86; tempo di protrombina $122 \%$; glicemia 64 ; azotemia $73 \%$; creatinina 0,80\%; AST 17; ALT 23; fosfatasi alcalina 59; bilirubinemia $0,30 \%$ (diretta 0,09\%; indiretta $0,21 \%$ ); sodio $135 \mathrm{mEq} / \mathrm{L}$; potassio $3,8 \mathrm{mEq} / \mathrm{L}$; calcio $8,5 \%$; ferro $57 \%$; proteine totali 6,3\%; albumina 3,3\%; acido urico $4,0 \%$; colesterolo totale 160\%; trigliceridi 205\%. Elettroforesi delle sieroproteine: albumina 49,9\%; alfa-1 6,0\%; alfa-2 10,4\%; beta-1 $6,4 \%$; beta-2 5,9\%; gamma 21,4\%; rapporto 1,0 .

Normali il CEA e gli altri marker tumorali (Ca 19.9, Ca 12.5, Ca 15.3, Ca 50). Urine normali.

Eelettrocardiogramma: anomalie della fase di ripolarizzazione. Radiografia del torace: ispessimento della trama broncovasale. Seni costofrenici liberi. Tenue sclerosi aortica. Slargamento del mediastino superiore di destra. Ecografia dell'addome: negativa. TAC total body: assenza di lesioni ripetitive.

Decorso: si è proceduto all'escissione delle masse tumorali delle regioni zigomatiche, in regime di Day Surgery presso l'Unità Operativa di Chirurgia Plastica, in due separate sedute. L'esame istologico (figg. 2-4) ha evidenziato: carcinoma a cellule squamose ben differenziato, ulcerato, infiltrante fino all'ipoderma, a focolai multipli.

L'intervento del collo ha richiesto un ricovero ospedaliero di alcuni giorni. In questa sede la neoplasia ha assunto le caratteristiche di un carcinoma destruente della regione latero-cervicale sinistra del collo, della regione sovraclaveare e del giugulo, con approfondimento sino ai vasi del collo (giugulare e carotide). Si è proceduto a un'ampia escissione e alla ricostruzione con lembo di rotazione, dopo emostasi accurata (fig. 5).

La degenza della paziente si è svolta unicamente in reparto medico, per fornire un'ottimale assistenza e per non aggravare lo scompenso psichico.

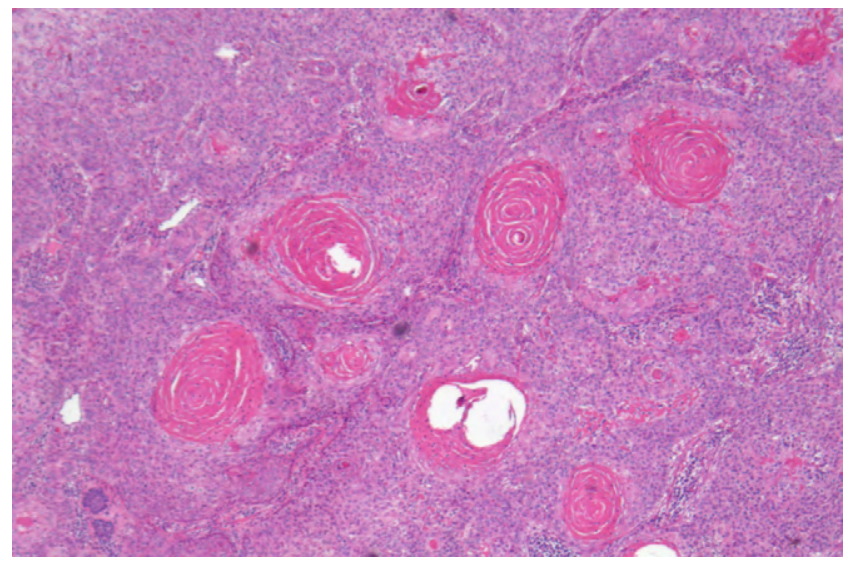

Figura 2 Carcinoma a cellule squamose ben differenziato, ulcerato, infiltrante fino all'ipoderma, a focolai multipli a piccolo ingrandimento. 


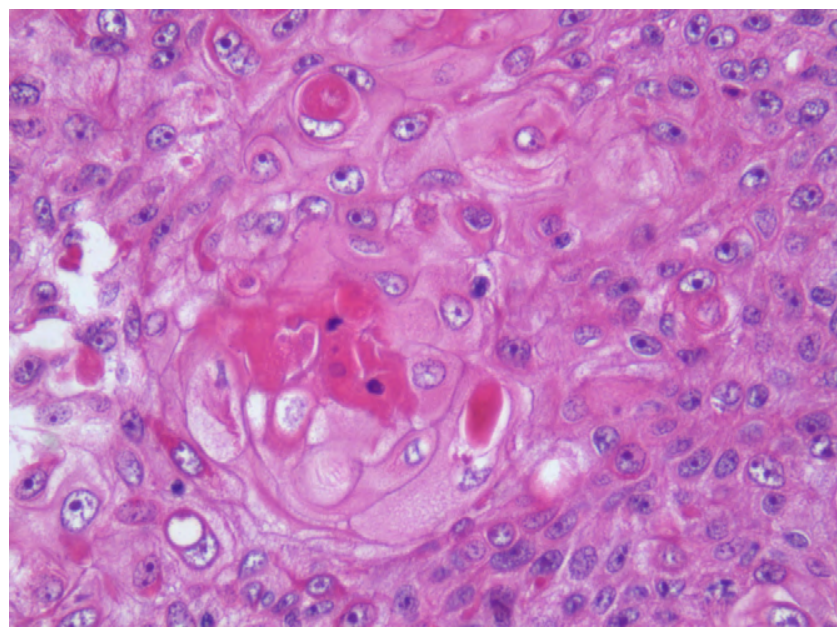

Figura 3 Carcinoma a cellule squamose ben differenziato, ulcerato, infiltrante fino all'ipoderma, a focolai multipli a maggiore ingrandimento.

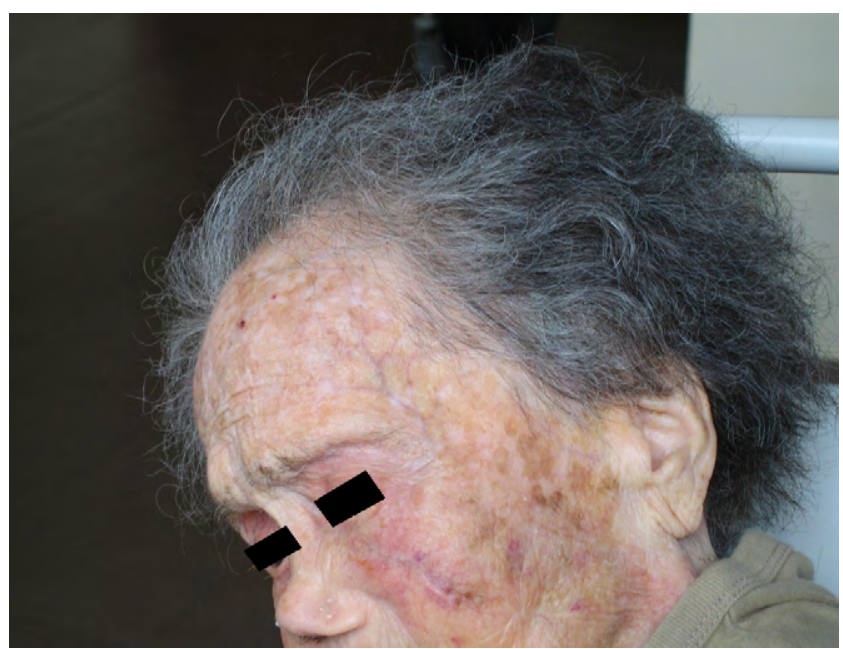

Figura 4 Dopo escissione della massa tumorale dello zigomo.

La scala psicometrica SPMSQ (Short Portable Mental Status Questionnaire) ha configurato una demenza grave. Erano inoltre presenti: scarsa capacità di ritenere le informazioni nella memoria a breve termine, facile affaticabilità, tendenza a riprodurre false preoccupazioni. Sul piano comportamentale, occasionalmente si osservavano riso immotivato, alternanza di atteggiamenti di conformità e atteggiamenti resistenti. Sul piano umorale, erano presenti sintomi ansiosi e sintomi depressivi, con abulia e mancanza di interessi.

Una degenza brevissima in ospedale, della durata di qualche giorno, seguita dall'immediato ritorno in Residenza Sanitaria Assistenziale, ha permesso un buon recupero; la paziente è stata reinserita nella vita di gruppo e ha ripreso il programma riabilitativo.

Sarà necessario un attento follow-up per monitorare sul piano clinico e strumentale il rischio di metastasi.

È stato ottenuto il consenso informato da parte dei familiari della paziente.

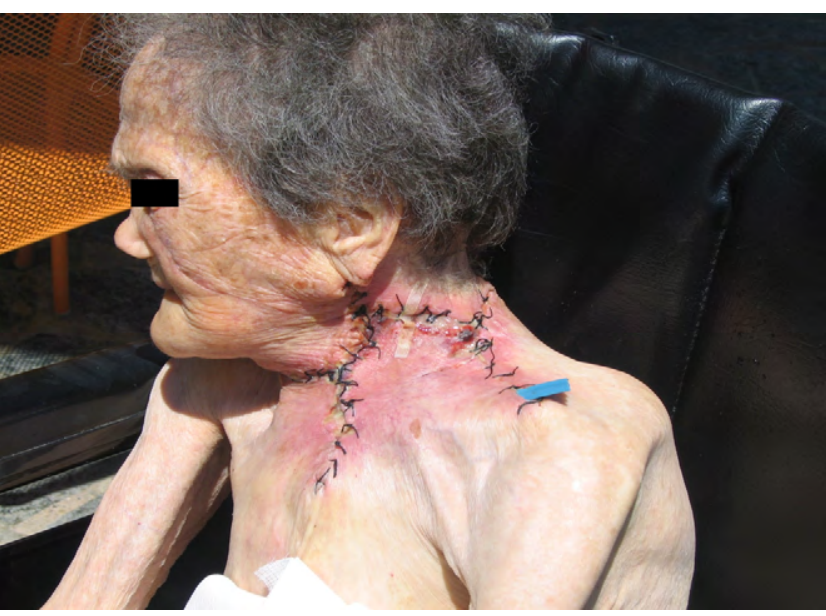

Figura 5 Dopo escissione della massa tumorale del collo.

\section{Discussione}

Il caso clinico descritto presenta vari spunti critici. Caratteristici erano la localizzazione del carcinoma, che ha interessato bilateralmente le regioni zigomatiche e il collo, l'età della paziente, ultranovantenne, la condizione psichica di demenza di grado elevato [6,7], il ricovero prima in Day Surgery e poi, brevissimo, di alcuni giorni, in ambiente medico, che ha consentito di provvedere, senza alcuna degenza in Chirurgia, alla gestione medica dell'atto chirurgico fornendo continuità assistenziale a una paziente con grave deterioramento cognitivo. In particolare, il caso esposto esprime ancora una volta la "complessità" [8-10] di cui si deve far carico la Medicina moderna, incentrata sul paziente, a differenza della Evidence-Base Medicine. La complessità rende la Medicina una scienza probabilistica, con un elevato rischio di errore per la necessità, da una parte, di assicurare il processo decisionale medico e, dall'altra, per il fatto di non poter adottare conoscenze ben definite.

\section{Conflitto di interesse}

Gli autori dichiarano di essere esenti da conflitto di interessi.

\section{Bibliografia}

[1] Cerimele D, Satta R. Carcinoma spinocellulare. In Giannetti A (a cura di). Trattato di dermatologia. Padova: Piccin, 2002. Vol IV, Cap 92: p. 1-21.

[2] Monti M. L'ulcera cutanea. Milano: Ambrosiana; 2000. p. 388.

[3] Braun-Falco O, Plewing G, Wolff HH, Winkelman RK. Dermatologia. Milano: Springer; 2002. p. 1462-89.

[4] Mazzeo F. Trattato di clinica e terapia chirurgica. Napoli: G. De Nicola; 2001. p. 1089.

[5] Sedda AF, Rossi G, Cipriani C. Brachiterapia dermatologica per la cura dei carcinomi della pelle. Energia Ambiente Innovazioni 2007;4:1-8.

[6] Fratiglioni L, De Ronchi D, Agüero-Torres H. Worldwide prevalence and incidence of dementia. Drugs Aging 1999;15(5): 365-75.

[7] De Ronchi D, Fratiglioni L, Rucci P, Paternicò A, Graziani S, Dalmonte $E$. The effect of education on dementia occurrence in 
an Italian population with middle to high socioeconomic status. Neurology 1998;50(5):1231-8.

[8] Nardi R, Fabbri T, Belmonte G, Leandri P, Mazzetti M, Pasquale $A$, et al. Medicina interna, paziente complesso, evidence based medicine e le non evidenze. Ital J Med 2009;3(4):191-200.
[9] Wilson T, Holt T, Greenhalgh T. Complexity science: complexity and clinical care. BMJ 2001;323(7314):685-8.

[10] Agency for Healthcare Research and Quality (AHRQ). Definition: complex patient, Funding Opportunity Announcement (FOA). Technical Assistance Conference. Call October 15, 2007. 